\title{
Norois
}

Environnement, aménagement, société

\section{SPOT5 : un outil pertinent pour le suivi du trait de côte?}

SPOT5 as a relevant tool for shoreline monitoring?

Iwan Le Berre, Alain Hénaff, Thomas Devogèle, Ariane Mascret et Franck Wenzel

\section{(2) OpenEdition}

1 Journals

\section{Édition électronique}

URL : https://journals.openedition.org/norois/378

DOI : $10.4000 /$ norois.378

ISBN : 978-2-7535-1544-4

ISSN : $1760-8546$

\section{Éditeur}

Presses universitaires de Rennes

\section{Édition imprimée}

Date de publication : 1 septembre 2005

Pagination : 23-35

ISBN : 978-2-7535-0218-5

ISSN : 0029-182X

\section{Référence électronique}

Iwan Le Berre, Alain Hénaff, Thomas Devogèle, Ariane Mascret et Franck Wenzel, « SPOT5 : un outil pertinent pour le suivi du trait de côte? », Norois [En ligne], 196 | 2005/3, mis en ligne le 15 décembre 2008, consulté le 13 janvier 2022. URL : http://journals.openedition.org/norois/378 ; DOI : https:// doi.org/10.4000/norois.378

Ce document a été généré automatiquement le 13 janvier 2022.

(c) Tous droits réservés 


\title{
SPOT5 : un outil pertinent pour le suivi du trait de côte?
}

\author{
SPOT5 as a relevant tool for shoreline monitoring? \\ Iwan Le Berre, Alain Hénaff, Thomas Devogèle, Ariane Mascret et Franck \\ Wenzel
}

\section{NOTE DE L'ÉDITEUR}

Cet article a été reçu le 18 mars 2005 et définitivement accepté le 10 octobre 2005.

Cet article est extrait du rapport réalisé en septembre 2004 par le laboratoire Géomer (I. Le Berre, A. Hénaff, J. Giraudet) associé au Centre d'Etudes Techniques Maritimes et Fluviales (F. Wenzel), dans le cadre de la convention établie entre le CNRS et le METLTM/DDE29.

Les données Shom sont exploitées dans le cadre de la convention Shom/UBO/CNRS/INSU

$n^{\circ}$ E50/2002. Les extraits de la BD ORTHO IGN ont été obtenus dans le cadre de la convention établie entre le CNRS et le METLTM/DDE29.

1 En milieu littoral, la mise en œuvre de bases d'information géographique est souvent confrontée à la juxtaposition de deux référentiels, terrestre et marin, dont la compatibilité n'est pas immédiate tant du point de vue de la délimitation des limites physiographiques que de celui des niveaux hypsométriques de référence (Cuq, 2000). C'est pourquoi le Comité technique du Groupe littoral du Conseil national de l'information géographique (CNIG) recommande la détermination d'une méthodologie nationale de définition d'un Référentiel Géographique Littoral ou RGL (CNIG, 2003; Robin et al., 2005). C'est, entre autre, dans ce contexte que se situe l'appel d'offre CNESIFEN $^{1}$ du 5 août 2002 relatif au suivi du littoral à grande échelle par imagerie SPOT5.

2 Le projet présenté par le laboratoire Géomer dans le cadre de cet appel à proposition visait à tester les possibilités de SPOT5 pour la cartographie de l'estran, du trait de côte et de l'occupation du littoral dans une perspective commune de contribution à la cartographie synthétique de l'environnement littoral (Le Berre et al., 2004). Seule la 
partie relative au trait de côte est exposée dans cet article. Elle a pour but d'évaluer le potentiel du capteur haute précision du satellite SPOT5 pour la délimitation d'un trait de côte de référence permettant de répondre à la fois à des besoins scientifiques relatifs au suivi de la dynamique du littoral et à des besoins opérationnels concernant la protection du littoral contre l'érosion marine, notamment pour la définition de la politique d'aménagement des ouvrages de protection contre la mer. D'un point de vue opérationnel, l'intérêt d'un tel référentiel se situe à plusieurs niveaux :

- le suivi de la cinématique littorale et du « linéaire côtier soumis à l'érosion », un des indicateurs perçus comme pertinents pour l'évaluation des politiques publiques au regard de la Loi Littoral ;

- la caractérisation du trait de côte, tant du point de vue de la nature du littoral que de celui de son artificialisation ;

- d'une manière générale, la production de statistiques territoriales sur le trait de côte homogènes au niveau national (type CORINE Érosion Côtière).

\section{Définition du trait de côte géomorphologique et problématique}

3 Limite de référence indéniable en tant que séparation linéaire entre deux domaines, terrestre et marin, le trait de côte est pourtant une notion ambiguë tant le passage de l'un vers l'autre est progressif dans l'espace et mobile dans le temps. Une synthèse de la littérature, établie par M. Robin (2002), a ainsi mis en évidence plus d'une douzaine de limites de référence d'usage courant sur le littoral, parmi lesquelles plusieurs peuvent être considérées en tant que trait de côte: ados de plage, ligne de crête supratidale, ligne exceptionnelle des eaux marines, etc. Parmi les nombreuses définitions existantes, nous avons retenu les références suivantes :

- Groupe de travail Shom-Ifremer (Allain et al., 2000), Géographie maritime, limites physiographiques, trait de côte : « Limite conventionnelle du domaine maritime au voisinage de la laisse de pleine mer pour les côtes de France Atlantique et Manche. Le long des côtes méditerranéennes, le trait de côte est défini comme le niveau maximal hivernal. Sa définition théorique pourrait être, soit la limite des plus hautes mers astronomiques, soit la limite des niveaux extrêmes de pleine mer d'une période de retour de dix ans. »

-J.-P. Pinot (1998), trait de côte : « Ligne qui marque la limite jusqu'à laquelle peuvent parvenir les eaux marines. Le trait de côte proprement dit est la limite la plus extrême que puissent atteindre les eaux marines, c'est-à-dire l'extrémité du jet de rive lors des fortes tempêtes survenant aux plus hautes mers de vives eaux.[...]. Le trait de côte n'est pas constamment visible sur le terrain par une discontinuité dans la topographie ou dans la couverture végétale. »

Ces définitions, proches l'une de l'autre, décrivent le trait de côte comme la limite maximale atteinte par la mer sur la côte (dans des conditions météorologiques normales ou paroxysmales). On peut constater que cette conception du trait de côte rejoint à peu de chose près celle du Domaine Publique Maritime (DPM), et que la définition théorique proposée par le Groupe de travail Shom-Ifremer sur les niveaux extrêmes correspondrait plus à une approche de type "risques de submersion ». Or c'est finalement la limite des plus hautes marées astronomiques qui a été retenue par le groupe de travail «Littoral» du CNIG en tant que «limite physique où la terre s'arrête » (CNIG, 2004). Cependant, ce type de définition est peu opérationnel dans un 
objectif de délimitation homogène du trait de côte, car elle relève de l'observation directe. Sur le terrain, la méthode du piquetage ${ }^{2}$ apparaît peu efficace et très lourde à mettre en oeuvre, si ce n'est sur des portions très restreintes du littoral, en raison de l'importance du personnel et de la coordination nécessaires à sa mise en œuvre. La délimitation à partir de photographies aériennes ou d'imagerie satellitaire impose quant à elle la correspondance des horaires de prise de vue, et donc des passages des aéronefs ou des satellites, avec celles de la marée. Dès lors, comment appréhender cette limite? Cette question placée au cœur de la réflexion menée sur la définition des données de référence spécifiques à la zone côtière par le Groupe de travail « littoral » du CNIG a donné lieu à la définition du « trait de côte géomorphologique » :

- CNIG (2003), géographie de la zone côtière, limites physiographiques, limite géomorphologique du trait de côte : "Lieu de discontinuité morphologique caractéristique de la limite probable atteinte par la mer sur la côte. Exemples : talus d'érosion, pied de dune, limite de végétation de la plage, laisse des pleines mers de vives eaux exceptionnelles, bermes de tempête... Cette limite morphologique présentant un intérêt pour le suivi de l'évolution physique des côtes. "

5 Ainsi, en proposant des critères de délimitation d'ordre morphologiques, sédimentaires et botaniques, le problème de la marée est écarté. D'un point de vue pratique, il reste encore à arrêter une méthodologie résultant de la pertinence et de l'adéquation de critères de délimitation avec un support de délimitation, au regard des objectifs à atteindre :

- produire une donnée de référence homogène et continue au niveau national ;

- permettre l'évaluation de la cinématique côtière et la production de statistiques territoriales.

\section{Matériel et méthodes}

Le projet s'appuie sur l'expérience acquise par Géomer, composante brestoise de LETG (UMR 6554 du CNRS), dans le domaine de la géomorphologie et de l'aménagement du littoral et dans celui de la production et du traitement de l'information géographique au sein de SIG. Les données de référence pour la validation des résultats sont extraites des bases d'information géographique développées au laboratoire sur le littoral finistérien ou ont été fournies par le ministère de l'Équipement via le CETMEF et la DDE du Finistère, partenaires de ce projet. La comparaison des différents traits de côte produits et acquis est réalisée par l'IRENav à l'aide de méthodes et d'outils originaux, basés sur la distance linéaire de Fréchet, développés par cet institut.

\section{L'image SPOT5}

7 Le projet a bénéficié de la programmation du satellite SPOT5 pour l'acquisition d'une image au cours d'une basse mer de vive-eau sur le littoral nord-ouest finistérien. Une image en mode multispectral (THR + XS) à 2,5 $\mathrm{m}$ de résolution a été acquise le 17 avril 2003, lors de la basse mer. Elle a été livrée au laboratoire Géomer au début de l'été 2003. Les conditions météorologiques et atmosphériques (anticyclone de $1025 \mathrm{hPa}$ ), l'heure de passage du satellite qui coïncidait assez étroitement avec le moment de la basse mer (moins d'une heure de décalage), ainsi que le coefficient de la marée (114) ont permis 
l'acquisition d'une image de très bonne qualité, montrant notamment l'estran dans sa quasi-intégralité (fig. 1).

Figure 1 : Extrait de l'image SPOT 5 acquise le 17 avril 2003, lors de la basse mer (secteur situé entre l'Aber Wrac'h et l'Aber Benoît). Le trait de côte et le zéro de la carte marine reportés sur l'image montrent qu'elle couvre presque intégralement la zone intertidale.

Extract of the SPOT 5 image of April 2003 the 17th, during a spring low-tide (area located between the Aber Wrac'h and the Aber Benoit). The shoreline and the theoretical lower spring tide level (zero of the marine chart) are overlaid on the image showing that the whole tidal zone is visible.

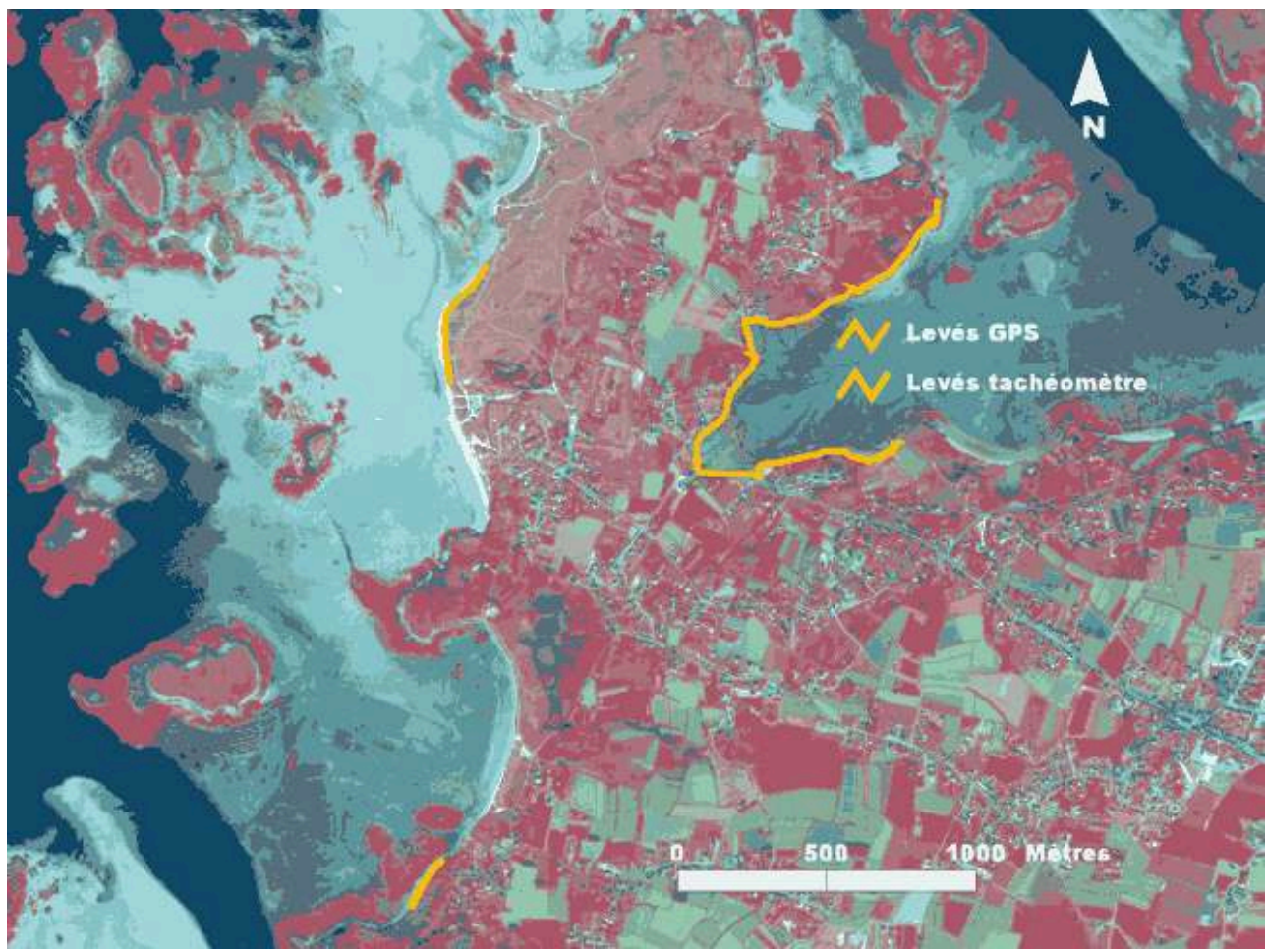

\section{Secteur d'étude}

La zone d'application retenue pour la comparaison des traits de côte se situe dans deux secteurs du nord Finistère (fig. 2). Le secteur des Abers, qui est constitué de portions de falaises meubles, de côtes rocheuses, d'ensembles dunaires et de vastes estrans, ainsi que de deux estuaires : l'Aber Benoît et l'Aber Wrac'h. Le secteur de Plougonvelin est quant à lui composé de falaises rocheuses élevées et d'une côte sableuse fortement artificialisée (le Trez Hir). Ces secteurs ont été choisis du fait (1) de leur représentativité des différents types de côtes de la région, (2) de la disponibilité des données de référence et (3) de la présence sur le terrain de l'équipe dans la période encadrant l'acquisition de l'image. 
Figure 2 : Localisation de la zone d'étude et des vues présentées dans les figures 3 à 7 Location of the study site and of the figures 3 to 7

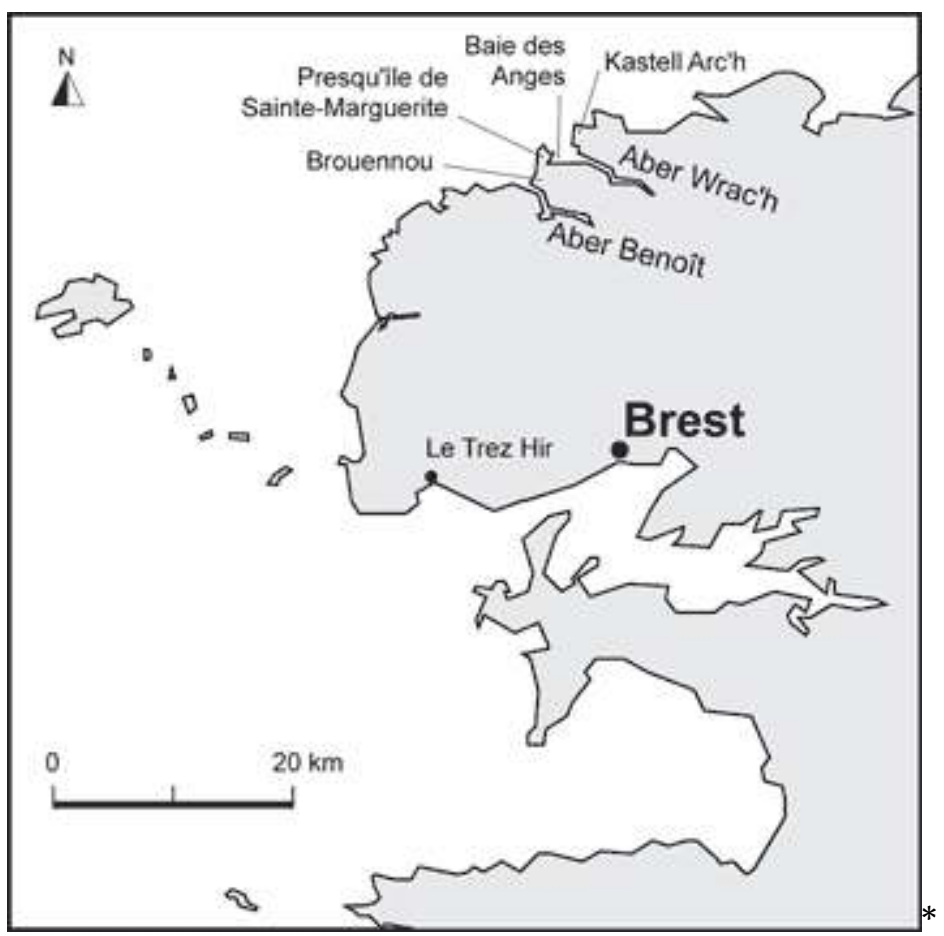

\section{Méthodes}

La méthode employée s'appuie sur des levés topographiques, sur l'interprétation de l'image SPOT5 et de la BDOrtho IGN, puis sur l'analyse comparative des différents traits de côte ainsi produits.

\section{Terrain}

Des levés topographiques détaillés de sections du trait de côte ont été réalisés sur le terrain dans la perspective de comparer les tracés obtenus à ceux issus de l'interprétation de l'image SPOT5 et de l'orthophotographie. Ces levés ont été effectués dans la presqu'île de Sainte-Marguerite en Landeda le jour du passage du satellite. Une section d'environ $200 \mathrm{~m}$ de falaises vives verticales érodées dans des formations quaternaires meubles dont le retrait exhume une plate-forme d'érosion littorale fossile a été levée sur la grève de Brouennou. Un second levé a été effectué à SainteMarguerite, le long d'un secteur d'environ $440 \mathrm{~m}$ d'une accumulation sableuse littorale constituée d'une plage adossée à un massif dunaire en accrétion ou tout au moins stabilisé. Enfin, une série de levés d'une longueur totale d'environ $3000 \mathrm{~m}$ a été réalisée dans la baie des Anges à l'entrée de l'Aber Wrac'h sur des portions de côte soit artificialisée, soit constituée de falaise basse végétalisée, soit constituée par un cordon de galet (fig. 3). Dans les secteurs de falaises, les deux lignes de référence définissant généralement le trait de côte, à savoir le pied de falaise (Bonnot-Courtois et Levasseur, 2003) et le rebord sommital de la partie érodée du versant (Robin, 2002), ont été mesurées mais c'est cette dernière qui a finalement été retenue pour la comparaison (voir les critères de délimitation présentés dans la partie résultats). Dans le cas du secteur sableux, seul le contact entre la limite de la végétation dunaire (oyat) a été pris 
en considération pour la cartographie du trait de côte (Robin, 2002). Sur le secteur artificialisé c'est la base des ouvrages qui a été mesurée. Les levés ont été exécutés (à Brouennou et à Saint-Marguerite) à l'aide d'un tachéomètre laser (Leica TCR 303) ou bien (baie des Anges) à l'aide d'un GPS différentiel Trimble 5800 qui fournissent l'un et l'autre une précision de mesure d'ordre centimétrique. Dans tous les cas, la position de la station a été déterminée à l'aide de points de calage géoréférencés et les mesures topographiques effectuées correspondent au relevé des points d'inflexion du tracé en plan du trait de côte. La distance entre les points mesurés varie donc en fonction de la complexité du tracé de chacun des traits de côte levés (tableau 1).

Figure 3 : Localisation des levés du trait de côte au tachéomètre (à Brouennou sur falaise meuble au sud, à Sainte-Margherite sur dune au nord)

Location of shoreline tacheometer surveys (Brouennou cliffs in the south, Sainte-Marguerite sand dunes in the north)

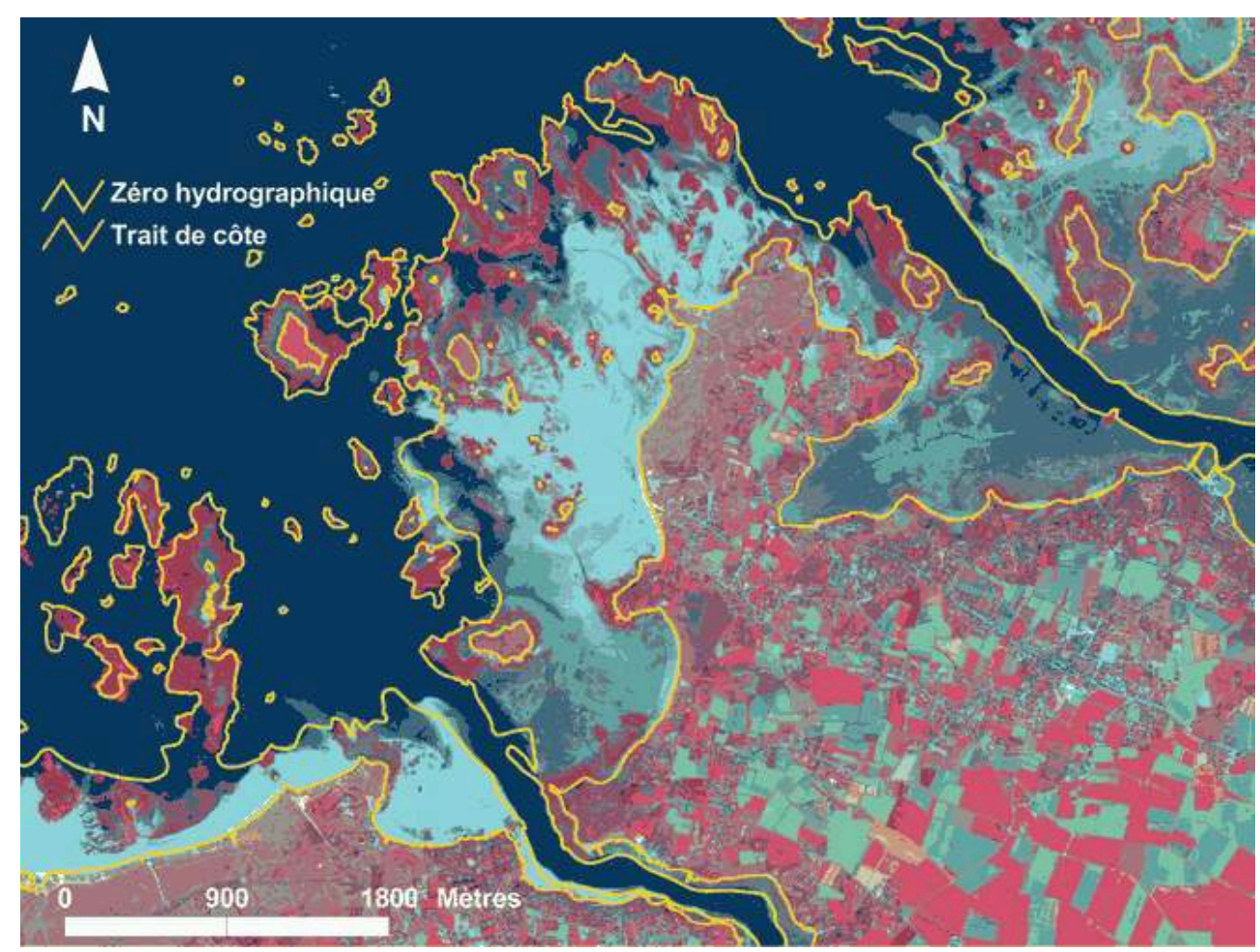

Tableau 1 : Spécifications des levés topographiques Caracteristics of the topographic surveys

\begin{tabular}{|l|c|c|}
\hline Levé & $\begin{array}{c}\text { Distance moyenne } \\
\text { entre les mesures (en m) }\end{array}$ & Écart type (en m) \\
\hline Falaises de Brouennou (rebord sommital) & 6,7 & 4,4 \\
Falaises de Brouennou (pied de falaise) & 6,6 & 5,1 \\
Dunes de Sainte-Marguerite & 9,7 & 5,7 \\
Cordon de galet baie des Anges & 5,8 & 2,3 \\
Falaise végétalisée baie des Anges & 8,2 & 4,9 \\
Grève baie des Anges & 6,8 & 4,6 \\
Ouvrages côtiers baie des Anges & 11,4 & 10,1 \\
\hline
\end{tabular}




\section{Interprétation de l'image SPOT5 et de l'orthophotographie}

11 L'image SPOT5 tout comme la BD Ortho IGN sont traitées par interprétation assistée par ordinateur afin d'étudier, de manière fine, les possibilités de délimitation et de caractérisation du trait de côte. L'image SPOT5 a fait l'objet de deux numérisations séparées, la première à $1 / 6000$ qui constitue une échelle confortable pour l'interprétation de l'image, la seconde à $1 / 1500$ qui constitue l'échelle à partir de laquelle les pixels deviennent nettement visibles et commencent à constituer une gêne pour l'interprétation de l'image. La BD Ortho pour sa part a été numérisée à $1 / 1500$, échelle de confort pour son interprétation. Chaque numérisation aboutit à la production de couches d'information géographique vectorielles linéaires créées sous ArcView 3.2. L'évaluation du potentiel de SPOT5 comparativement à celui de l'orthophotographie repose, d'une part, sur les critères proposés en synthèse de l'étude de C. Bonnot-Courtois et J.-E. Levasseur $(2002,2003)$ qui représentent les plus forts indices de présomption de présence de la limite atteinte par la mer sur la côte; et, d'autre part, sur les critères morphologiques, sédimentaires et botaniques visibles sur image et pouvant présenter un intérêt au regard des objectifs à atteindre. Sur les différents types de côtes rencontrés dans la zone d'étude, le critère le plus pertinent est retenu pour évaluer la précision de la limite cartographiée pour produire la donnée " trait de côte géomorphologique » (tableau 2). Cette délimitation permet ainsi :

- de proposer une gamme d'échelles de restitution au delà de laquelle la donnée produite n'est plus utilisable ;

- d'estimer la précision moyenne, à travers l'erreur commise lors de la saisie sur la position du trait de côte ;

- d'établir une densité moyenne de points et une vitesse moyenne de numérisation de la donnée, estimée d'après le temps de photo-interprétation sur la zone test ;

- et, enfin, d'établir un rapport entre les linéaires produits par chacun des supports.

\section{Comparaison quantitative des traits de côte}

12 Les traits de côte ainsi interprétés sont ensuite comparés aux données de référence collectées lors des levés topographiques. Les points des extrémités des segments constituant ces traits de côte sont exportés dans un fichier texte à l'aide d'Arc/Info. Ces lignes sont traitées par un exécutable développé en C++. Elles sont suréchantillonnées pour être comparées à l'aide de mesures dérivées de la distance de Fréchet (Devogèle, 2000). Chaque mesure fournit une estimation décimétrique de l'écart métrique entre une ligne de référence et une ligne saisie.

13 Ainsi, les objectifs visés sont-ils, d'une part, d'évaluer qualitativement et quantitativement l'information issue de l'image satellitaire, de la positionner techniquement et financièrement par rapport à celle issue de la photographie aérienne, et, d'autre part, de proposer une méthodologie de suivi du trait de côte éprouvée à partir de ces différents types de données.

\section{Résultats}

Les résultats de la cartographie du trait de côte géomorphologique sont présentés par type de côte. Les critères de délimitation employés sont d'abord définis et discutés. Ces résultats ainsi qu'une information sur la précision de la donnée produite sont ensuite 
synthétisés dans le tableau 2. Une comparaison des résultats obtenus lors de cette délimitation sur chacun des deux supports utilisés est ensuite proposée.

\section{Critères de délimitation sur les côtes rocheuses et falaises maritimes : falaises de roches dures}

Quatre critères potentiels, morpho-sédimentaires et botaniques, sont identifiés pour délimiter le trait de côte géomorphologique sur les côtes de roches dures de la zone d'étude.

- Rupture de pente du profil et dépoli de la base : ce critère géomorphologique est d'efficacité et d'appréhension aléatoire, indépendamment de la méthode ou du support de délimitation, car trop assujetti, d'une part, à l'exposition de la côte (il est généralement plus marqué en mode abrité) et, d'autre part, aux caractéristiques du site : nature de la roche, stratification, pendage des couches géologiques, etc. Dans un travail de photo-interprétation, tant sur SPOT5 que sur la BD ORTHO ${ }^{\circ}$, ce critère est inutilisable car, en l'absence de la troisième dimension (nous ne disposions pas pour cette étude de couples d'images SPOT5, ni des couples de photographies aériennes à l'origine de la BD ORTHO ${ }^{\circ}$ ) et dans la gamme des résolutions considérées (les produits stéréoscopiques issus de SPOT5 offrent une précision altimétrique seulement décamétrique), il n'est pas ou que très rarement observable.

- Pied de falaise : lorsque l'escarpement d'une falaise de roche dure est assez abrupt et que son pied est déblayé sous l'action de la mer (falaises vives), cette limite, même si elle se situe en dessous du niveau des plus hautes eaux, peut être pertinente pour la délimitation des domaines terrestre et marin ainsi que pour le suivi du recul de la falaise. En revanche, les décalages liés à l'inclinaison de certaines prises de vues, tant sur SPOT5 que sur la BD $\mathrm{ORTHO}^{\circ}$, et aux redressements géométriques subséquents posent le problème de la photointerprétation de cette limite dès lors que le haut de falaise surplombe et masque (ombrage) l'escarpement et le pied de falaise.

- La limite supérieure du lichen marin noir(Verrucaria maura) est le meilleur indicateur botanique de la limite supérieure des pleines mers de vives eaux en mode abrité. Il perd de sa précision et de sa possibilité de distinction lorsque l'exposition et l'anfractuosité de la roche augmentent et quand la côte s'échancre. Ce critère de délimitation n'est quasiment pas observable sur l'image SPOT5 du fait de sa trop faible résolution et de son faible contraste. Sur la BD ORTHO ${ }^{\circ}$, on a parfois un assez bon contraste entre la limite supérieure de la bande de lichen et la roche, mais le manque de régularité radiométrique ne permet pas de l'utiliser comme limite du trait de côte géomorphologique.

- La limite de la végétation terrestre en haut de falaise semble plus adaptée pour la délimitation du trait de côte géomorphologique. Elle représente le meilleur compromis entre des critères (a) pouvant être représentatifs de la limite maximale atteinte par la mer sur la côte et/ou pertinents pour le suivi de l'évolution physique des côtes mais qui ne sont observables que ponctuellement sur SPOT5 et/ou la BD ORTHO ${ }^{\circ}$; et (b) l'exigence de continuité et d'homogénéité de la donnée à produire, car il peut être identifié partout (de par son contraste), indépendamment du fait que la falaise soit vive ou stabilisée, plongeante ou présentant une plate-forme d'érosion, actuelle ou héritée, constituée de roches dures, de roches sédimentaires ou de formations meubles. Toutefois, les évolutions de cette limite peuvent avoir d'autres causes que les variations de la limite de la mer : stress anthropiques, pollutions et variations du milieu d'une manière générale, ce qui impose une connaissance locale pour son interprétation. 
Malgré ces réserves, c'est ce dernier critère qui est retenu pour l'établissement de la cartographie de la zone d'étude.

\section{Critères de délimitation sur les côtes meubles : plages adossées à un massif dunaire}

- Pour la délimitation du trait de côte géomorphologique sur les plages adossées à un massif dunaire deux critères morpho-sédimentaires peuvent être utilisés selon que la plage est en érosion ou stabilisée et/ou en accrétion. Sur les plages en érosion ces critères sont un profil concave et la présence de micro-falaises d'érosion en pied de dune ; sur les plages stables ou en accrétion, il s'agit du profil convexe et de la berme de haut de plage. Cependant, outre le fait que ces critères sont soumis aux variations saisonnières des profils de plage, et donc peu stables pour établir la limite atteinte par la mer, il n'est pas possible de détecter un quelconque relief de l'ados dunaire, tant sur l'image SPOT5 que sur l'orthophotographie ;

- Le critère botanique de délimitation est représenté ici par la limite inférieure d'extension de l'Oyat continental avant qu'il ne soit remplacé par le Chiendent des sables en haut de plage. Dans la gamme des résolutions considérées, il n'est cependant pas possible de différencier les espèces végétales.

17 Le critère retenu est donc la limite inférieure de la végétation dunaire, fortement contrastée tant sur l'image SPOT5 que sur la BD ORTHO ${ }^{\circ}$. Cependant cette limite présente de nombreuses discontinuités de par le cisaillement des dunes sous l'action des écoulements ou des sentiers d'accès à la plage. Ces dépressions rendent difficiles l'interprétation de la limite des plus hautes mers. Dans ce cas, le trait de côte est prolongé dans son alignement global. Un léger traitement de l'image par simple amélioration des contrastes permet cependant de rendre cette limite plus visible.

\section{Critères de délimitation sur les côtes basses : schorre}

Sur les schorres, la micro-topographie du haut-schorre et la limite entre le schorre supérieur et la végétation terrestre sont les critères géomorphologique et botanique potentiels de délimitation du trait de côte géomorphologique.

- La micro-topographie du haut-schorre correspond à des différences de contraste entre la végétation terrestre, le schorre supérieur et la slikke qui peuvent localement donner à l'image SPOT5 une impression de relief que l'on ne ressent pas sur l'orthophotographie. Cela n'en fait pas pour autant un critère exploitable car ces contrastes topographiques sont très atténués, voire inexistants lorsque le haut du schorre avoisine une végétation herbacée.

- La limite entre le schorre supérieur et la végétation terrestre est le critère identifié. Elle est peu contrastée sur l'orthophotographie, mais pleinement exploitable de par sa résolution submétrique. Toutefois les ombrages portés par les arbres en diminuent la précision. Cette limite est, par contre, relativement contrastée sur l'image SPOT5 ce qui en fait un critère satisfaisant de délimitation et de suivi des évolutions du schorre.

Bien que des confusions locales soient toujours possibles qui appellent un contrôle avec l'orthophotographie, c'est cette limite qui est retenue pour la cartographie du trait de côte géomorphologique. 


\section{Critères de délimitation sur les côtes artificialisées}

20 artificialisées est la limite des aménagements de défense contre la mer, des installations portuaires et des endiguements des zones basses.

Pour les installations portuaires, l'image SPOT5 ne permet pas une appréhension et une délimitation correcte des zones portuaires dès lors que leurs dimensions sont faibles. Sur l'orthophotographie littorale (fig. 5) deux parkings, une cale et un émissaire sont visibles alors qu'ils sont peu ou pas discernables sur l'image SPOT5 ce qui conduit à des erreurs de délimitation présentées sur la figure 4. Il est encore plus difficile d'appréhender les aménagements de défense des fronts de mer aménagés sur SPOT5 car la résolution de 2,5 $\mathrm{m}$ est souvent supérieure à l'épaisseur des ouvrages, notamment lorsqu'il s'agit de murs ou de perrés (murs de soutènement). Sur les figures 6 et 7 , l'orthophotographie montre un front de mer constitué d'un espace vert et d'un parking protégés par un perré à peine discernable au niveau du parking. Cet aménagement (parking + perré) n'est pas identifiable sur l'image SPOT5 entraînant une erreur de délimitation. De même, les enrochements, de largeur pourtant plus importante, restent peu ou pas visibles sur l'image, alors qu'ils peuvent être identifiés sur l'orthophotographie. Enfin, les endiguements des zones basses, généralement couverts de végétation ne sont pas non plus visibles sur l'image SPOT5.

En définitive, le seul support de délimitation ne peut suffire à appréhender l'artificialisation du littoral. Les ouvrages ne peuvent pratiquement pas être identifiés à l'aide de l'image SPOT5 et l'exploitation de l'orthophotographie est également sujette au risque d'erreur d'interprétation, qui requiert alors un contrôle systématique sur le terrain.

Figures 4 et 5 : Numérisation des ouvrages portuaires à Kastell Ac'h en Plouguerneau d'après l'image SPOT 5. La superposition du trait de côte numérisé à la BD ORTHO ${ }^{\circledR}$ montre que les parkings, la cale et l'émissaire difficiles à discerner sur l'image SPOT 5 conduisent à des erreurs d'interprétation.

Harbour equipments in Plouguerneau digitized from the SPOT 5 image and overlaid with the BD $O R T H O{ }^{\circledR}$. The difficulty to identify the car parks, the slip and the outlet on the SPOT 5 image lead to misinterpretations.
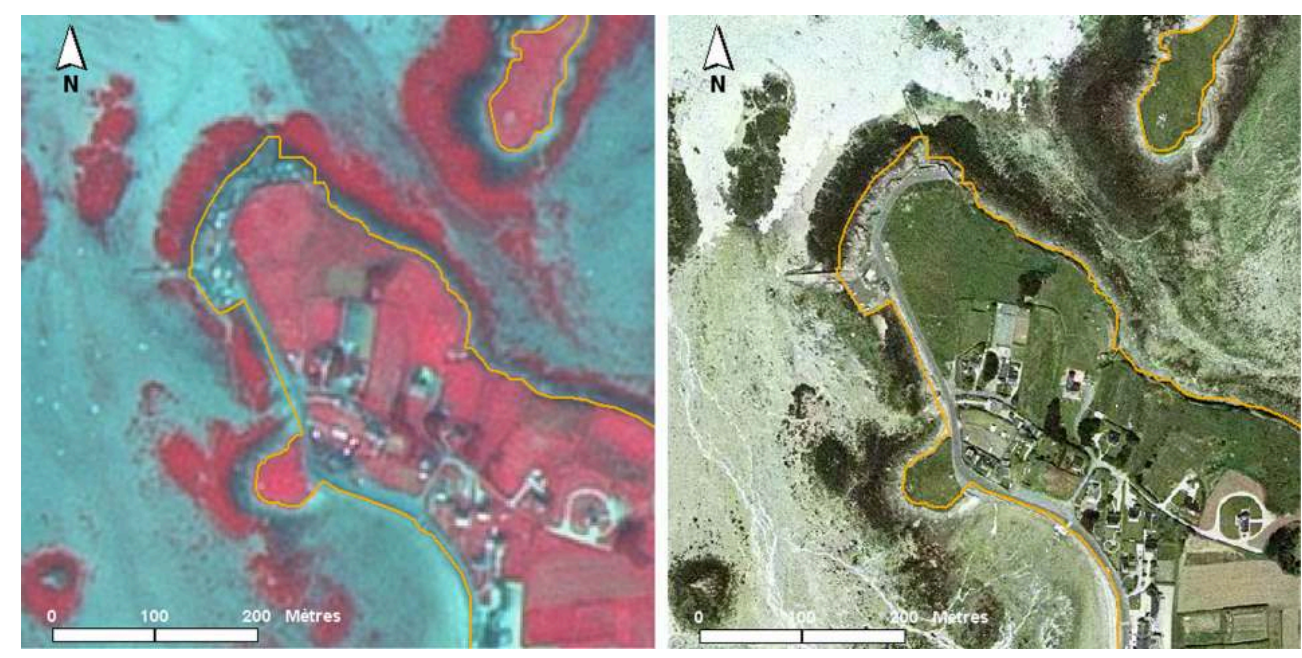
Figures 6 et 7 : Les ouvrages de défense contre la mer du Trez-Hir à Plougonvelin, peu distincts sur SPOT 5 conduit à une erreur d'interprétation dans la délimitation du trait de côte visible lorsqu'il est superposé à la BD ORTHO® (au nord est de l'image)

Figures 6 and 7: The poor distinction of Plougonvelin's sea walls on SPOT 5 may lead to misinterpretation of shoreline as shown when overlaid on the $B D O R T H O{ }^{\circledR}$ (see the north east part of the pictures)
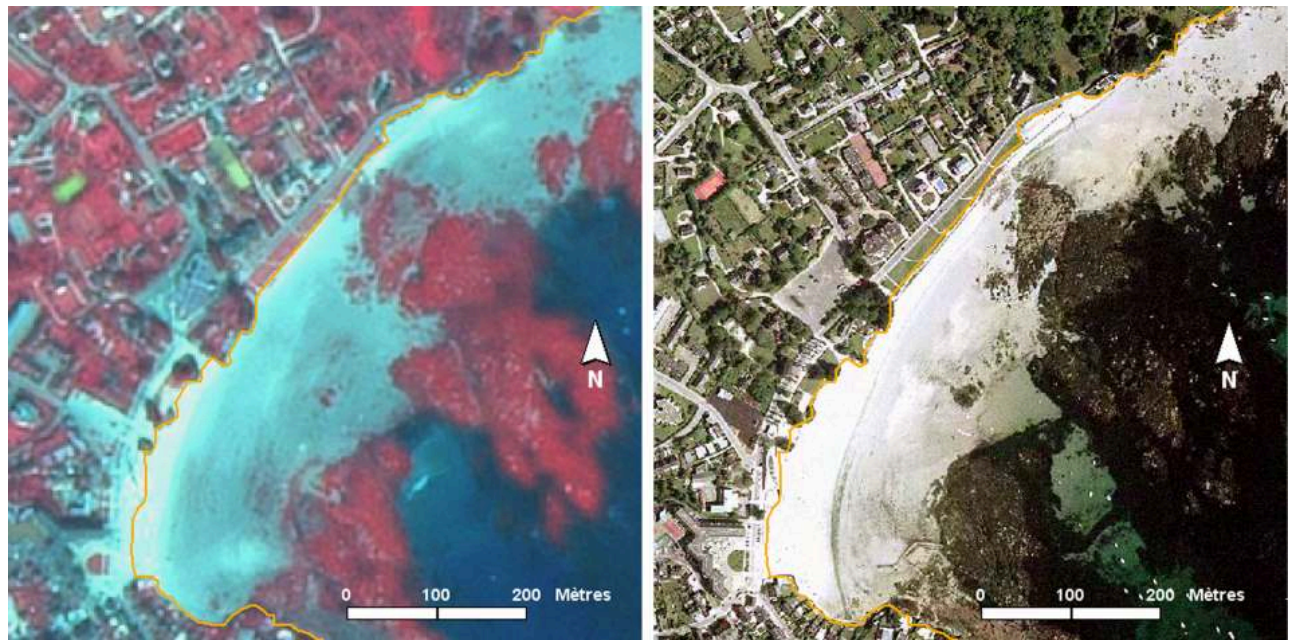

\section{Caractérisation de la nature du trait de côte}

Sur ce point, les conclusions sont très proches de celles relatives à la description des ouvrages côtiers. Elles sont synthétisées dans le tableau 3.

L'expérimentation réalisée montre que, dans la plupart des cas, la résolution spatiale de l'image SPOT5 est insuffisante pour pouvoir caractériser sans ambiguïté la nature du trait de côte. L'appui de l'orthophotographie et des investigations sur le terrain s'avèrent ici indispensables.

\section{Comparaison quantitative de la précision de numérisation}

L'écart entre deux lignes peut être caractérisé par un grand nombre de mesures. Elles évaluent, pour la plupart, l'écart métrique entre ces lignes. Cependant d'autres mesures peuvent s'avérer utiles pour comparer la forme (sinuosité, courbure, direction, etc.). Pour cette étude, seules des mesures sur l'écart métrique entre deux lignes ont été réalisées. Elles qualifient la précision de numérisation en comparant les traits de côte photo-interprétés d'après l'image SPOT5 (deux interprétations aux échelles de 1/6 000 et de $1 / 1500$ ) et la BD ORTHO (à l'échelle de $1 / 1500$ ) avec des traits de côte de référence acquis par tachéomètrie ou par GPS différentiel. 
Tableau 2 : Synthèse de la pertinence des critères de délimitation du trait de côte géomorphologique et précision de la donnée produite

Synthetic table of the relevance of the indicators choosen to define the geomorphologic shoreline and precision of the data produced

\begin{tabular}{|c|c|c|c|}
\hline \multicolumn{4}{|c|}{$\begin{array}{l}\text { FALAISES ROCHES DURES } \\
\end{array}$} \\
\hline \multicolumn{2}{|c|}{\begin{tabular}{|l|l|} 
Pertinence des critères de délimitation & \\
\end{tabular}} & SPOT5 & BD Ortho 2000 \\
\hline \multirow[t]{2}{*}{ Critères botaniques } & $\begin{array}{l}\text { Limite supérieure du lichen } \\
\text { marin noir incrustant } \\
\text { Verrucaria maura }\end{array}$ & -- & - \\
\hline & $\begin{array}{l}\text { Limite de la végétation terrestre } \\
\text { en haut de falaise }\end{array}$ & ++ & ++ \\
\hline \multirow[t]{2}{*}{$\begin{array}{l}\text { Critères morphologiques } \\
\text { et sédimentologiques }\end{array}$} & \begin{tabular}{|l|l|} 
Rupture de pente éventuelle \\
et possible dépoli à la base
\end{tabular} & -- & -- \\
\hline & \begin{tabular}{|l|l} 
Pied de falaise & \\
\end{tabular} & - & - \\
\hline \multicolumn{4}{|c|}{ Critère retenu : limite de la végétation terrestre en haut de falaise } \\
\hline \multicolumn{2}{|c|}{\begin{tabular}{|l|l} 
Échelle de numérisation &
\end{tabular}} & \multicolumn{2}{|c|}{$1 / 2000$} \\
\hline \multicolumn{2}{|l|}{ Échelle de restitution } & Du $1 / 5000$ au $1 / 25000$ & Du $1 / 2500$ au $1 / 10000$ \\
\hline \multicolumn{2}{|l|}{ Précision moyenne } & $<20 \mathrm{~m}$ & $<10 \mathrm{~m}$ \\
\hline \multicolumn{2}{|l|}{ Densité moyenne de points } & 160 points $/ \mathrm{km}$ & 285 points/km \\
\hline \multicolumn{2}{|c|}{ Vitesse moyenne de numérisation } & $23 \mathrm{~km} / \mathrm{h}$ & $11 \mathrm{~km} / \mathrm{h}$ \\
\hline \multicolumn{4}{|c|}{ PLAGE ADOSSÉE À UN MASSIF DUNAIRE } \\
\hline \multicolumn{2}{|c|}{\begin{tabular}{|l|l} 
Pertinence des critères de délimitation &
\end{tabular}} & SPOT5 & BD Ortho 2000 \\
\hline \multirow[t]{2}{*}{ Critères botaniques } & \begin{tabular}{|l|l|} 
Limite entre le Chiendent \\
des sables et l'Oyat continental
\end{tabular} & -- & -- \\
\hline & \begin{tabular}{|l|l} 
Limite inférieure de \\
la végétation dunaire
\end{tabular} & ++ & ++ \\
\hline \multirow[t]{2}{*}{$\begin{array}{l}\text { Critères morphologiques } \\
\text { et sédimentologiques }\end{array}$} & $\begin{array}{l}\text { En érosion : profil concave, } \\
\text { micro-falaise d'érosion } \\
\text { en pied de dune }\end{array}$ & -- & -- \\
\hline & $\begin{array}{l}\text { En progression ou stable: } \\
\text { profil convexe, berme de haut } \\
\text { de plage }\end{array}$ & -- & -- \\
\hline \multicolumn{4}{|c|}{ Critère retenu : limite inférieure de la végétation dunaire } \\
\hline \multicolumn{2}{|c|}{ Échelle de numérisation } & \multicolumn{2}{|c|}{$1 / 2000$} \\
\hline \multicolumn{2}{|l|}{ Échelle de restitution } & Du $1 / 5000$ au $1 / 25000$ & Du $1 / 2500$ au $1 / 10000$ \\
\hline \multicolumn{2}{|l|}{ Précision moyenne } & $<10 \mathrm{~m}$ & $<5 \mathrm{~m}$ \\
\hline \multicolumn{2}{|l|}{ Densité moyenne de points } & 50 points $/ \mathrm{km}$ & 90 points $/ \mathrm{km}$ \\
\hline \multicolumn{2}{|c|}{ Vitesse moyenne de numérisation } & $30 \mathrm{~km} / \mathrm{h}$ & $20 \mathrm{~km} / \mathrm{h}$ \\
\hline \multicolumn{4}{|c|}{ SCHORRE } \\
\hline \multicolumn{2}{|c|}{ Pertinence des critères de délimitation } & SPOT5 & BD Ortho 2000 \\
\hline Critères botaniques & $\begin{array}{l}\text { Limite entre la végétation du } \\
\text { schorre supérieur et } \\
\text { la végétation terrestre }\end{array}$ & + & + \\
\hline $\begin{array}{l}\text { Critères morphologiques } \\
\text { et sédimentologiques }\end{array}$ & $\begin{array}{l}\text { Micro-topographie } \\
\text { du haut schorre }\end{array}$ & - & -- \\
\hline \multicolumn{4}{|c|}{ Critère retenu : limite entre la végétation du schorre supérieur et la végétation terrestre } \\
\hline \multicolumn{2}{|c|}{\begin{tabular}{|l|l} 
Échelle de numérisation &
\end{tabular}} & \multicolumn{2}{|c|}{$1 / 2000$} \\
\hline \multicolumn{2}{|l|}{ Échelle de restitution } & Du $1 / 5000$ au $1 / 25000$ & Du $1 / 5000$ au $1 / 25000$ \\
\hline \multicolumn{2}{|l|}{ Précision moyenne } & $<15 \mathrm{~m}$ & $<15 \mathrm{~m}$ \\
\hline \multicolumn{2}{|c|}{ Densité moyenne de points } & 130 points $/ \mathrm{km}$ & 155 points/km \\
\hline Vitesse moyenne de numéris & tion & $25 \mathrm{~km} / \mathrm{h}$ & $25 \mathrm{~km} / \mathrm{h}$ \\
\hline
\end{tabular}


Tableau 3 : Comparaison de SPOT5 et de la BD ORTHO ${ }^{\circledR}$ IGN 2000 pour la caractérisation du trait de côte SPOT5 and BD ORTHO® IGN 2000 comparison for the identification of the shoreline

\begin{tabular}{|c|c|c|}
\hline $\begin{array}{l}\text { Nature du trait } \\
\text { de côte }\end{array}$ & $\begin{array}{c}\text { Image SPOT5 } \\
\text { Composite couleur à } 2,50 \mathrm{~m} \text { de résolution } \\
\text { Acquise le } 17 \text { avril } 2003 \text { à Midi }\end{array}$ & $\begin{array}{l}\text { BD Ortho" } 2000 \\
\text { Mosaique couleur à } 0,50 \mathrm{~m} \text { de résolutions patizle } \\
\text { Acquise en juin } 2000 \text { à heures variables }\end{array}$ \\
\hline Schorre & $\begin{array}{l}\text { + Visible etcontrasté; } \\
\text { - Dans la partie haute, confusion possible avec } \\
\text { la végétation herbacée de la berge et avec l'ombrage } \\
\text { porté par les arbres. }\end{array}$ & $\begin{array}{l}\text { - Visible, bien que peu contrasté; } \\
\text { - Dans la partie haute, confusion possible avec la } \\
\text { végétation herbacée de la berge et avec l'ombrage } \\
\text { porté par les arbres. }\end{array}$ \\
\hline Dune & $\begin{array}{l}\text { + Trait de côte bien distinct lorsque } \\
\text { la dune est végètalisēe; } \\
\text { - Délimitation moins évidente dans le sable nu. }\end{array}$ & $\begin{array}{l}\text { ++ Trait de côte parfaitement distinct } \\
\text { brsque ba dune est végètalisée ; } \\
\text { - Moins visible dans le sable nu. }\end{array}$ \\
\hline $\begin{array}{l}\text { Gôte basse et } \\
\text { falaise rocheuse } \\
\text { ou me uble }\end{array}$ & $\begin{array}{l}\text { - La hauteur et la nature des falaises ne peuwent être } \\
\text { identifiées. La résolution n'est pas assez fine, la prise } \\
\text { de vue semi-verticale interdt d'avoir une idée de la } \\
\text { hauteur de la côte, les effets d'ombrages empêchent } \\
\text { une délimitation. L'utilisation d'informations complé- } \\
\text { mentaires provenant de sources telles que la carte } \\
\text { topographique ou le terrain sont indispensables } \\
\text { pour caractériser le littoral. }\end{array}$ & $\begin{array}{l}\text { La hauteur approximative et la nature des falaises } \\
\text { peuvent être identiffíées, mais nécessitent impérative } \\
\text { ment une validation sur le terrain etiou l'analyse } \\
\text { stéréoscopique des couples de photographies } \\
\text { aériennes (non disponibles pour cette étude). }\end{array}$ \\
\hline $\begin{array}{l}\text { Ouwrage } \\
\text { portuaire }\end{array}$ & $\begin{array}{l}\text { - Peu visibles, à l'exception des oumrages les plus } \\
\text { massifs dont on distingue les contours. }\end{array}$ & $\begin{array}{l}\text { ++ Parfaitement identifrables et génè ralement } \\
\text { caractérisables. }\end{array}$ \\
\hline $\begin{array}{l}\text { Ouvrage de } \\
\text { prodectioncontre } \\
\text { la mer }\end{array}$ & $\begin{array}{l}\text { - La majeure partie des ouvrages ne peut être distinguée } \\
\text { sur l'image, à l'exception des plus importants etiou } \\
\text { de ceux qui présentent un fort contraste avec leur } \\
\text { enwironnement immédiat; } \\
\text { - Une bonne connaissance du terrain reste en tout cas } \\
\text { indispensable pour l'identification des ouvrages. } \\
\text { - Leur nature ne peut pas être caractérisée. }\end{array}$ & $\begin{array}{l}\text { + La plupart des ouvrages peuvent être } \\
\text { généralement distingués et caractér risés à partir } \\
\text { de la photographie aérienne; } \\
\text { - En raison de leur faible étendue ou de leur } \\
\text { apparence sur l'orthophoto, certains ouwrages se } \\
\text { confondent cependant avec leur environnement } \\
\text { immédiat; } \\
\text { - L'appui du terrain est donc essentiel pour la } \\
\text { production d'une donnée de qualité. }\end{array}$ \\
\hline $\begin{array}{l}\text { Bâtiment, } \\
\text { ouvrage de voirie }\end{array}$ & $\begin{array}{l}\text { - Peu visible, à l'exception des ouwrages les plus massifs } \\
\text { dont on distingue les contours. }\end{array}$ & $\begin{array}{l}\text { ++ Parfaitement identifrables et génè ralement } \\
\text { caractérisables. }\end{array}$ \\
\hline $\begin{array}{l}\text { Lithral awec } \\
\text { vêgetation haute }\end{array}$ & $\begin{array}{l}\text { - Difficulté de délimitation liée à l'effet d'ombre } \\
\text { de la végétation selon la position du satellite; } \\
\text { - Lorsque l'estran est également végétalisé, } \\
\text { le contraste est faible entre les deux types de } \\
\text { végétation et la délimitation du trait de côte } \\
\text { peu évidente. }\end{array}$ & $\begin{array}{l}\text { - Difficulté de délimitation liée aux ombres portées } \\
\text { par la végétation : le trait de côte ne peut être } \\
\text { identifié précisément. }\end{array}$ \\
\hline
\end{tabular}

26 Pour comparer la géométrie de deux objets ponctuels, la distance euclidienne s'impose. Par contre, pour deux objets linéaires, plusieurs distances peuvent être employées ( $\mathrm{T}$. Devogele, 2000). Ces distances reflètent soit l'écart maximum entre les deux lignes (distances maximales), soit l'écart moyen entre les deux lignes (distances moyennes). Ces deux types de distances sont complémentaires. Les distances moyennes fournissent des informations sur les différences de position entre les deux lignes dans leur globalité. Les distances maximales indiquent l'écart le plus important. L'utilisation de distances minimales n'est pas pertinente dans ce contexte.

Devogèle (2000) a montré que la distance de Fréchet entre deux lignes orientées était la distance maximale la plus adaptée pour ce type de mesure. Cette distance s'appuie sur la propriété suivante: toute polyligne orientée est équivalente à une application continue $\mathrm{f}:[\mathrm{a}, \mathrm{b}] \rightarrow \mathrm{V}$ où $\mathrm{a}, \mathrm{b} \in \mathcal{R}, \mathrm{a}<\mathrm{b}$ et $\mathrm{V}$ est l'espace vectoriel. La distance de Fréchet $\left(\mathrm{d}_{\mathrm{F}}\right)$ est la suivante :

$$
\begin{aligned}
& \text { Soit } f:\left[a, a^{\prime}\right] \rightarrow V \text { et } g:\left[b, b^{\prime}\right] \rightarrow V \text { deux polylignes et }\|\| \text { la norme usuelle, } \\
& \qquad d F(f, g)=\inf _{\substack{\alpha:[0,1] \rightarrow\left[a, a^{\prime}\right] \\
\beta:[0,1] \rightarrow\left[b, b^{\prime}\right]}} \max _{t \in[0,1]}\|f((\alpha(t))-g(\beta(t)))\|
\end{aligned}
$$

Une illustration intuitive de la distance de Fréchet est la suivante : un maître et son chien suivent deux chemins (f et $g$ ). Ils avancent ou s'arrêtent à volonté, indépendamment l'un de l'autre, mais ils ne peuvent pas revenir sur leurs pas. La distance de Fréchet entre ces deux chemins est la longueur minimale de la laisse qui permet de réaliser un cheminement de concert satisfaisant ces conditions.

La distance de Fréchet a l'avantage d'être calculée uniquement à partir de couples de points qui peuvent être mis en correspondance visuellement (points homologues). La 
distance de Fréchet est donc très proche d'une distance maximale "visuelle » entre deux lignes. Hélas, cette distance a l'inconvénient d'être complexe à programmer. Dans ce même article (Devogele, 2000), il a été proposé une distance approchée définie dans H. Alt et M. Godau (1995) qui permet de calculer cette distance dans des temps raisonnables: la distance de Fréchet discrète. L'erreur d'approximation peut néanmoins être contrôlée par suréchantillonnage des lignes. De plus, de nouvelles distances dérivées de cette distance ont été présentées :

- la distance de Fréchet discrète partielle qui procure une mesure de l'écart maximal entre une ligne et la partie homologue d'une ligne de plus grande emprise. Cette distance a été utilisée pour comparer les lignes issues de la photo interprétation et les levés exécutés à l'aide du tachéomètre laser ;

- la distance de Fréchet discrète moyenne qui fournit un écart moyen entre deux lignes orientées, en calculant la moyenne des distances des points déterminés comme homologues ;

- la distance de Fréchet discrète partielle moyenne combine les deux premières mesures. Elle renvoie une mesure de l'écart moyen entre une ligne et la partie homologue d'une ligne de plus grande emprise.

\begin{tabular}{|c|c|c|c|c|c|c|}
\hline \multicolumn{7}{|c|}{ Trait de côte de référence (levé tachéomètre ou GPS) } \\
\hline $\begin{array}{c}\text { Photo- } \\
\text { interprétation }\end{array}$ & $\begin{array}{c}\text { Rebord sommital } \\
\text { de falaise } \\
\text { (Brouennou) }\end{array}$ & $\begin{array}{c}\text { Falaise } \\
\text { végétalisée (baie } \\
\text { des Anges) }\end{array}$ & $\begin{array}{c}\text { Dune (Sainte- } \\
\text { Marguerite) }\end{array}$ & $\begin{array}{c}\text { Grève (baie } \\
\text { des Anges) }\end{array}$ & $\begin{array}{c}\text { Cordon littoral } \\
\text { (baie des Anges) }\end{array}$ & $\begin{array}{c}\text { Ouvrages côtiers } \\
\text { (baie des Anges) }\end{array}$ \\
\hline SPOT5, 1/6000 & $3,34(6,07)$ & $4,54(11,98)$ & $2,25(6,08)$ & $0,96(3,90)$ & $2,01(4,18)$ & $2,37(6,26)$ \\
SPOT5, $1 / 1500$ & $1,62(3,78)$ & $2,77(10,84)$ & $1,17(3,79)$ & $1,22(5,20)$ & $1,45(3,39)$ & $3,58(14,01)$ \\
BD ORTHO & $1,03(3,10)$ & $2,8(7,25)$ & $2,1(8,64)$ & $1,41(5,38)$ & $1,82(5,08)$ & $1,77(7,37)$ \\
$1 / 1500$ & & & & & & \\
\hline
\end{tabular}

Tableau 4 : Distances en mètres entre les lignes saisies et les lignes de référence, distance de Fréchet discrète partielle moyenne suivie de la distance de Fréchet discrète partielle entre parenthèses (précision décimétrique)

Distances in meter between digitized lines and reference lines, the average partial discrete Fréchet distance followed by the partial discrete Fréchet distance between round brackets

Sur la zone de falaise vive et verticale, les résultats du tableau 4 montrent qu'en moyenne la précision de photo-interprétation réalisée à partir de la $\mathrm{BD}$ ORTHO ${ }^{\circ}$ est légèrement meilleure que celle issue de SPOT5. Pour la zone de falaise végétalisée, les résultats sont relativement proches et montrent que la végétation constitue manifestement une contrainte pour une numérisation précise.

31 En zone dunaire, les traits de côte issus de SPOT5 sont légèrement meilleurs que ceux issus de la BD ORTHO . La simple évolution qu'a connue la ligne de rivage en haut de plage entre le moment de la prise de l'orthophotographie (juin 2000) et celui de l'image SPOT5 et du levé tachéométrique (avril 2003) permettent certainement d'expliquer la meilleure précision obtenue ici à partir de l'image SPOT5. Elle démontre cependant l'intérêt de l'imagerie SPOT5 pour le suivi à court terme de l'évolution des littoraux meubles.

De même, les meilleurs résultats obtenus à l'aide de SPOT5 pour l'interprétation du cordon de galet et de la zone de grève doivent être interprétés avec prudence et à la lumière d'une possible évolution de trait de côte ou de la végétation qui la recouvre entre les deux prises de vues.

En zone artificielle l'orthophotographie permet l'obtention de résultats sensiblement meilleurs ( $25 \%$ d'amélioration). Le faible résultat obtenu pour le trait de côte numérisé à 1/1 500 d'après SPOT5 est essentiellement lié à une différence dans l'interprétation et dans le choix de l'objet à numériser, qui est également à l'origine de la forte distance de 
Fréchet discrète (écart maximal) dans les trois cas. Cette valeur témoigne de la difficulté à identifier précisément les ouvrages côtiers.

Ainsi, globalement, sur cette zone d'étude, si l'on excepte la numérisation effectuée au $6000^{\mathrm{e}}$ d'après l'image SPOT5, les photo-interprétations de SPOT5 ont une précision du même ordre de grandeur que celles de la BD ORTHO ${ }^{\circ}$.

\section{Bilan}

À l'issue des expériences réalisées, il apparaît d'une manière générale que la limite interprétée entre les domaines terrestre et marin, tant sur l'image satellite SPOT5 que sur l'orthophotographie littorale mériterait peut-être plutôt l'appellation de trait de côte «botanique » que "géomorphologique » de par les critères exploitables dans la gamme des résolutions considérées.

Quoiqu'il en soit, si SPOT5 présente des potentialités intéressantes en termes de précision de délimitation, la caractérisation du trait de côte reste moins bonne qu'avec l'orthophotographie. La résolution de l'image SPOT5 et les incertitudes de délimitation liées notamment aux effets d'ombre, à la confusion entre la végétation terrestre et la végétation intertidale, rendent peu évidente une caractérisation du trait de côte. C'est particulièrement évident en ce qui concerne les ouvrages côtiers qui peuvent rarement être identifiés et encore moins caractérisés sur l'image, à l'exception des ouvrages les plus importants.

37 L'utilisation de SPOT5 paraît néanmoins appropriée pour la cartographie du trait de côte, notamment dans certains secteurs dunaires sur lesquels le contraste entre la partie terrestre et le domaine marin, souligné par la végétation, peut être déterminé et délimité relativement aisément. Compte tenu de la mobilité de ce type de côte, une voie potentiellement intéressante peut être explorée. D'après les tests de numérisation et la comparaison des mesures effectuées in situ, il apparaît que ces secteurs de côte peuvent être cartographiés avec une précision satisfaisante au regard de celle obtenue à partir de l'orthophotographie. L'image SPOT5 offre donc un certain nombre de perspectives en matière de suivi à court terme de l'évolution du trait de côte qui s'additionnent aux potentialités offertes par la répétitivité d'acquisition de données.

\section{BIBLIOGRAPHIE}

allain (S.), guillaumont (B.), LeVisage (C.), Loubersac (L.), populus (J.), 2000. - « Données géographiques de référence en domaine littoral », dans POPULUS (J.), LOUBERSAC (L.) (éd.), CoastGIS'99: Geomatics and coastal environment, Shom/Ifremer, p. 67-79.

ALT (H.), GODAU (M.), 1995 - « Computing the Fréchet distance between two polygonal curves », International Journal of Computational Geometry \& Applications, vol. 5, n 1-2, p. 75-91.

BONNOT-COURTOIS (C.), LEVASSEUR (J.-E.), 2002. - Reconnaissance de la limite terrestre du domaine maritime : intérêt et potentialités des critères morpho-sédimentaires et botaniques, Rapport CETMEF- 
ministère de l'Équipement, des Transports, de l'Aménagement du territoire, du Tourisme et de la Mer, [http://www.cetmef.equipement.gouv.fr].

BONNOT-COURToIs (C.), Levasseur (J.-E.), 2003. - « Recherche d'indicateurs "naturalistes" de la limite supérieure du domaine maritime » Cahiers Nantais, $n^{\circ}$ 59, p. 47-56.

CNIG, 2003. - Rapport final du Groupe de travail Littoral, Rapport Conseil national de l'information géographique, [http://www.cnig.gouv.fr].

CUQ (F.), 2000. - « Systèmes d'information géographique et gestion intégrée des zones côtières », dans Populus (J.), Loubersac (L.) (éd.), CoastGIS'99 : Geomatics and coastal environment, Shom/ Ifremer, p. 18-29.

DEVOGÈLE T., 2000. - « Mesure d'exactitude et processus de fusion à l'aide de la distance de Fréchet discrète ", Revue internationale de Géomatique, Numéro spécial Cassini 2000, Boursier (P.) (éd.), vol. 10, n 3-4, Éd. Hermès, p. 359-381.

LEBERRE (I.), HÉNAFF (A.), WENZEL (F.), GIRAUDET (J.), 2004. - Cartographie synthétique de l'environnement littoral du Finistère, exploitation de SPOT pour la cartographie de l'estran, du trait de côte et de l'occupation du littoral, Rapport final, Appel à proposition CNES/IFEN « Suivi du littoral par SPOT5 », laboratoire Géomer/Cetmef/DDE29, 108 p.

PINOT (J.-P.), 1998. - La gestion du littoral, Paris, Institut Océanographique, 2 vol., 670 p. ROBIN (M.), 2002. - « Télédétection et modélisation du trait de côte et de sa cinématique », dans BARON-YELLÈs (N.), GOELDNER-GIANELLA (L.), VELUT (S.) (dir.), Le littoral, regards, pratiques et savoirs, Paris, ENS, p. 95-115.

ROBIN (M.), MORSETTI (V.), PICHEREAU (D.), BABILLOT (P.), 2005. - « L'information géographique de référence en zone côtière : le cas français ", dans GOURMELON (F.), ROBIN (M.), SIG et environnement côtier, Traité IGAT, Éd. Hermès, p. 49-84.

\section{NOTES}

1. CNES : Centre national d'études spatiales ; IFEN : Institut français de l'environnement.

2. Méthode de délimitation, à l'aide de piquets, d'un terrain ou d'une limite par un arpenteurgéomètre. D'après J.-P. Pinot (1998), le DPM est défini « comme le terrain couvert par la mer calme lors des plus hautes mers possibles, plus précisément lors du plus fort flot du mois de Mars. Son extension peut être constatée officiellement par un piquetage opéré lors de cette pleine mer ».

\section{RÉSUMÉS}

Réalisée dans le cadre de l'appel d'offre CNES-IFEN d'août 2002 relatif au suivi du littoral à grande échelle par imagerie SPOT5, cette étude présente l'expérimentation réalisée pour la délimitation et la caractérisation du «trait de côte géomorphologique » tel que défini par le Conseil national de l'information géographique. Une comparaison des traits de côte issus de la photointerprétation de la BD ORTHO ${ }^{\circ}$ et d'une image SPOT5 avec des données de référence produites 
lors de levés topographiques sur le terrain est réalisée à l'aide de méthodes originales notamment basées sur la distance linéaire de Fréchet. Les résultats obtenus montrent que si SPOT5 présente des potentialités comparables à celles de la photographie aérienne pour la délimitation du trait de côte, sa résolution reste trop faible pour le caractériser sans ambiguïté. Cependant, notamment sur les secteurs dunaires où la végétation délimite nettement la partie terrestre et le domaine marin, la répétitivité de SPOT5 ouvre des perspectives intéressantes pour le suivi à court terme de l'évolution du trait de côte.

This paper gives a presentation of an experimentation of shoreline mapping implemented in the frame of the CNES-IFEN coastal zone monitoring with SPOT5 program. A comparison, based on original methods such as the Fréchet linear distance computation is made between (a) geomorphologic shorelines interpreted and digitized from orthophotographs and from a SPOT5 image and (b) reference data produced during topographic survey or extracted from the French mapping agency databases. Our experimentation shows comparable results for shoreline mapping, but not for shoreline identification where SPOT5 resolution is too low for an accurate distinction of coast types. On vegetated sand dunes especially, where shoreline is well demarcated, SPOT5 may offer interesting potentialities for frequent monitoring, thanks to its capability of regular data acquisition.

\section{INDEX}

Mots-clés : cartographie, SPOT5, suivi, trait de côte

Index géographique : Bretagne, Finistère

Keywords : mapping, monitoring, shoreline, SPOT5

\section{AUTEURS}

\section{IWAN LE BERRE}

Géomer - UMR 6554 LETG CNRS, Université de Bretagne Occidentale, Institut Universitaire Européen de la Mer, Technopôle Brest-Iroise, place Nicolas Copernic, 29280 Plouzané, France, iwan.leberre@univ-brest.fr

\section{ALAIN HÉNAFF}

Géomer - UMR 6554 LETG CNRS, Université de Bretagne Occidentale, Institut Universitaire Européen de la Mer, Technopôle Brest-Iroise, place Nicolas Copernic, 29280 Plouzané, France, alain.henaff@univ-brest.fr

\section{THOMAS DEVOGÈLE}

IRENav (Institut de Recherche de l'École Navale), BP 600, 29240 Brest Armées, France, devogele@ecole-navale.fr

\section{ARIANE MASCRET}

IRENav (Institut de Recherche de l'École Navale), BP 600, 29240 Brest Armées, France, mascret@ecole-navale.fr 


\section{FRANCK WENZEL}

CETMEF (Centre d'Étude Techniques Maritimes et Fluviales de l'Équipement), Technopôle BrestIroise, BP 5, 29280 Plouzané, France,

Frank.Wenzel@equipement.gouv.fr 